\title{
Model Reduction by Matching the Steady-State Response of Explicit Signal Generators
}

\author{
Giordano Scarciotti and Alessandro Astolfi
}

\begin{abstract}
Model reduction by moment matching for "interpolation signals" which do not have an implicit model, i.e. they do not satisfy a differential equation, is considered. Particular attention is devoted to discontinuous, possibly periodic, signals. The notion of moment is reformulated using an integral matrix equation. It is shown that, under specific conditions, the new definition and the one based on the Sylvester equation are equivalent. New parameterized families of models achieving moment matching are given. The results are illustrated by means of a numerical example.
\end{abstract}

\section{INTRODUCTION}

$\mathbf{T}$ HE model reduction problem can be informally formulated as the problem of finding a simplified description of a dynamical system, in specific operating conditions, preserving at the same time specific properties, e.g., stability. The model reduction problem has been addressed from several perspectives: exploiting Hankel operators [1], [2], [3]; the theory of balanced realizations [4], [5], [6]; the notion of moment matching [7], [8], [9], [10]. For an extensive list of references see the monograph [11]. In [12] a new interpretation of the moment matching problem has been given exploiting the center manifold theory. The first outcome of this interpretation is the ability to preserve some properties of the original system overcoming some of the drawbacks of the moment matching methods. The second outcome is the extension of the model reduction by moment matching theory to nonlinear systems, see e.g. [13], [14] and [15].

This paper generalizes the results of [12] with regards to the class of input signals considered. In [12] it has been shown that the moments of the system are in one-to-one relation with the eigenvalues of the dynamic matrix $S$ of the exogenous system $\dot{\omega}=S \omega$. Here, we consider input signals generated by a linear exogenous system represented in explicit form ${ }^{1}$, i.e. an implicit (differential) form may not exist. This direction of investigation is motivated by a large number of applications in which standard operating conditions are associated to non-continuous or non-differential input signals. For instance, power converters are controlled by means of pulse width modulated (PWM) signals or sawtooth signals, see e.g. [18], [19], [20], and [21]. To this end, a new integral equation to characterize the moments is given. Under specific assumptions, the equivalence of this new definition and the one based on the Sylvester equation given in [12] is proved.

G. Scarciotti is with the Dept. of Electrical and Electronic Engineering, Imperial College London, London, SW7 2AZ, UK, E-mail: gs3610@ic.ac.uk.

A. Astolfi is with the Dept. of Electrical and Electronic Engineering, Imperial College London, London, SW7 2AZ, UK and DICII, University of Rome "Tor Vergata", Via del Politecnico 1, 00133 Rome, Italy, E-mail: a.astolfi@ic.ac.uk. This work is partially supported by the EPSRC Programme Grant "Control For Energy and Sustainability" EP/G066477.

${ }^{1}$ The terminology is taken from [16], [17]. See also Definition 1.
Finally a new family of reduced order models is presented and connections with the family of models given in [12] are drawn.

The rest of the paper is organized as follows. In Section II we give a formal description of the problem addressed. In Section III the definition of moment is reformulated for explicit signal generators. In Section IV a family of reduced order models for linear systems is introduced. Finally Section V contains some concluding remarks.

A preliminary version of this paper has been submitted in [22]. Therein a detailed analysis of the relation between the "interpolation-based" and the "steady-state-based" descriptions of moment, which serves as an introduction for the present paper, is given. The novel contribution of the present paper consists in giving a new family of reduced order models and providing the technical details regarding the definition of moment which are missing in [22].

Notation. We use standard notation. $\mathbb{R}_{\geq 0}$ denotes the set of non-negative real numbers, $\mathbb{R}_{>0}$ denotes $\mathbb{R}_{\geq 0} \backslash\{0\}, \mathbb{C}_{<0}$ denotes the set of complex numbers with strictly negative real part and $\mathbb{C}_{\geq 0}$ denotes $\mathbb{C} \backslash \mathbb{C}_{<0}$. The symbol $I$ denotes the identity matrix, $\sigma(A)$ denotes the spectrum of the matrix $A \in \mathbb{R}^{n \times n}$ and $\|A\|$ indicates its induced Euclidean matrix norm. The symbol $\mathcal{L}(f(t))$ denotes the Laplace transform of the function $f(t)$ (provided that $f(t)$ is Laplace transformable) and, abusing the notation, $\sigma(\mathcal{L}(f(t)))$ denotes the set of poles of $\mathcal{L}(f(t))$. Given two functions, $f: Y \rightarrow Z$ and $g: X \rightarrow Y$, with $f \circ g: X \rightarrow Z$ we denote the composite function $(f \circ g)(x)=f(g(x))$ which maps all $x \in X$ to $f(g(x)) \in Z$.

\section{PRELIMINARIES}

\section{A. On the notion of moment}

In this section we recall the "steady-state-based" description of moment as introduced in [12], see also [22].

Consider a linear, single-input, single-output, continuous-time, system described by the equations

$$
\dot{x}=A x+B u, \quad y=C x,
$$

with $x(t) \in \mathbb{R}^{n}, u(t) \in \mathbb{R}, y(t) \in \mathbb{R}, A \in \mathbb{R}^{n \times n}, B \in$ $\mathbb{R}^{n \times 1}$ and $C \in \mathbb{R}^{1 \times n}$. Let $W(s)=C(s I-A)^{-1} B$ be the associated transfer function and assume that (1) is minimal, i.e. controllable and observable.

Theorem 1: [12] Consider system (1), $s_{i} \in \mathbb{C}$ and suppose $s_{i} \notin \sigma(A)$, for all $i=1, \ldots, \eta$, and $\sigma(A) \subset \mathbb{C}_{<0}$. Let $S \in \mathbb{R}^{\nu \times \nu}$ be any non-derogatory matrix with characteristic polynomial $p(s)=\prod_{i=1}^{\eta}\left(s-s_{i}\right)^{k_{i}}$, where $\nu=\sum_{i=1}^{\eta} k_{i}$. Consider the interconnection of system (1) with the system

$$
\dot{\omega}=S \omega, \quad u=L \omega
$$


with $L$ and $\omega(0)$ such that the triple $(L, S, \omega(0))$ is minimal. Then there exists a one-to-one relation between the moments ${ }^{2}$ $\eta_{0}\left(s_{1}\right), \ldots, \eta_{k_{1}-1}\left(s_{1}\right), \ldots, \eta_{0}\left(s_{\eta}\right), \ldots, \eta_{k_{\eta}-1}\left(s_{\eta}\right)$ and the steady-state response $C \Pi \omega$ of the output $y$ of such interconnected system, where $\Pi$ is the unique solution of the Sylvester equation

$$
A \Pi+B L=\Pi S .
$$

Remark 1: The minimality of $(L, S, \omega(0))$ is equivalent to the condition $\sigma\left(\mathcal{L}\left(L e^{S t} \omega(0)\right)\right)=\sigma(S)$ and it guarantees, together with the minimality of $(A, B, C)$, that all the modes of $\omega$ are excited and observable in the output.

The reduction technique based on this notion of moment consists in the interpolation of the steady-state response of the output of the system: a reduced order model is such that its steady-state response is equal to the steady-state response of the output of system (1) (provided it exists).

\section{B. Problem formulation}

The result described in Section II-A is based on the availability of a differential representation of the signal generator, namely equation (2). However, there are notable applications in which this may not be the case. For instance, the input of a dynamical system describing a power electronic device can often be a PWM wave which cannot be represented as the output of a system described by smooth differential equations. For this reason we introduce the following definition.

Definition 1: Let $x$, with $x(t) \in \mathbb{R}^{n}$, be the state of a dynamical system $\Sigma$. Let $u$, with $u(t) \in \mathbb{R}^{m}$, be the input of $\Sigma$. Let $t_{0}$ and $x_{0}=x\left(t_{0}\right)$ be the initial time and the initial state, respectively. If there exists a function $\phi: \mathbb{R} \times \mathbb{R} \times \mathbb{R}^{n} \times \mathbb{R}^{m} \rightarrow$ $\mathbb{R}^{n}$ such that

$$
x(t)=\phi\left(t, t_{0}, x_{0},\left.u\right|_{\left[t, t_{0}\right)}\right),
$$

for all $t \geq t_{0}$, we call equation (4) the representation in explicit form [16], or the explicit model, of $\Sigma$.

Assume $\phi\left(t, t_{0}, x_{0}, u\right)$ has a continuous derivative with respect to $t$ for every $t_{0}, x_{0}$ and $u$, and there exists a function $f$ : $\mathbb{R}^{n} \times \mathbb{R}^{m} \rightarrow \mathbb{R}^{n}$ continuous for each $t$ over $\mathbb{R}^{n} \times \mathbb{R}^{m}$ such that

$$
\dot{x}=f(x, u) .
$$

We call the differential equation (5) the representation in implicit form [17], or the implicit model, of $\Sigma$.

The goal of the paper is to extend the theory developed in [12] for linear signal generators which have an implicit model to signal generators in explicit form. Thus, consider

$$
\omega(t)=\Lambda\left(t, t_{0}\right) \omega_{0}, \quad u=L \omega,
$$

with $\Lambda\left(t, t_{0}\right) \in \mathbb{R}^{\nu \times \nu}$ such that $\Lambda\left(t_{0}, t_{0}\right)=I$. Note that this model provides a very general class of models which contains the implicit model (2), but that describes several other signal generators. For instance, any periodic signal can be described by (6) with the property

$$
\Lambda\left(t, t_{0}\right)=\Lambda\left(t-T, t_{0}\right), \quad t \geq T+t_{0},
$$

\footnotetext{
${ }^{2}$ The k-moment of system (1) at $s_{i}$ is $\eta_{k}\left(s_{i}\right)=\left.\frac{(-1)^{k}}{k !} \frac{d^{k} W(s)}{d s^{k}}\right|_{s=s_{i}}$.
}

where $T$ is the period of the signal $u$. Alternatively (6) can represent a signal generator described by a time-varying system of the form

$$
\dot{\omega}=S(t) \omega, \quad u=L \omega,
$$

in which case $\Lambda\left(t, t_{0}\right)$ is (with additional assumptions, e.g. the semigroup property) the transition matrix associated to (8) [23, Section 3].

Since the definition of moment given in the rest of the paper is based on the existence of the steady-state response of system (1) driven by (6), we need to introduce further hypotheses on the class of input signals (6).

Assumption 1: The vector $\omega(t)$ defined in equation (6) has a strictly proper Laplace transform with non-negative poles.

Assumption 1 is a standard condition for the existence of a well-defined steady-state response of the state of system (1) driven by (6) [24], [25], [26].

Assumption 2: The matrix valued function $\Lambda\left(t, t_{0}\right)$ is nonsingular for all $t \geq t_{0}$.

Assumption 2 is essential to have uniqueness of the solution $\omega(t)$ of (6). Note, in fact, that it is always satisfied by a generator of the form (2) and it is required for the uniqueness of the solution of system (8) (see e.g. [23]).

Assume now that there exists a set $\mathcal{T} \subset \mathbb{R}_{\geq 0}$ in which $\Lambda\left(t, t_{0}\right)$ is differentiable with respect to $t$ and consider the time-varying system described by the equation

$$
\dot{z}(t)=G(t)^{\top} z(t),
$$

with $G(t)=-\dot{\Lambda}\left(t, t_{0}\right) \Lambda\left(t, t_{0}\right)^{-1}$. Let $\Phi\left(t, t_{0}\right)$ be the transition matrix of system (9).

Assumption 3: The function $G(t)$ is piecewise continuous with respect to $t$. Moreover, there exist $T \geq t_{0}$ and a polynomial $p(t)$ such that $\left\|\Phi\left(t, t_{0}\right)^{\top}\right\| \leq p(t)$ for all $t \geq T$.

This last technical assumption guarantees that the norm of $z(t)$ in system (9) does not diverge to infinity exponentially [23, Section 29] and it is needed, as shown in the next section, to guarantee that the steady-state response ${ }^{3} x_{s}(t)$ of system (1) driven by (6) can be written as $x_{s}(t)=\Pi(t) \omega(t)$ for some matrix valued function $\Pi(t)$. Moreover, the piecewise continuity of $G(t)$ guarantees that the steady-state response is unique. Note that Assumption 3 is a generalization of the assumption (used in [12]) that $S$ in (2) is such that $\sigma(S) \subset \mathbb{C}_{\geq 0}$. In this case $G(t)=-S$ and if $\sigma(S) \subset \mathbb{C}_{\geq 0}$, the condition $\left\|e^{-S\left(t-t_{0}\right)}\right\| \leq p(t)$ holds trivially.

Remark 2: Assumptions 1, 2 and 3 are "mild" assumptions. For instance, they are satisfied by the general class of discontinuous periodic signals which are considered in the second part of the paper.

As already anticipated, among all the possible signals generated by an explicit model, we are particularly interested in periodic signals, which are generated by system (6) with the property (7) and its generalizations (see the next section).

\footnotetext{
${ }^{3} x_{s}(t) \in \mathbb{R}^{n}$ is the steady-state response of $x(t) \in \mathbb{R}^{n}$ if, for any $x\left(t_{0}\right)$, $\lim _{t \rightarrow+\infty} x(t)-x_{s}(t)=0$.
} 


\section{DEFINITION OF MOMENT}

In this section we give a definition of moment in the case in which the signal generator does not have an implicit model. We begin by showing that the interconnection of system (1) with the signal generator (6) possesses a steady-state response $x_{s}(t)$ described by the relation $x_{s}(t)=\Pi(t) \omega(t)$ for some matrix valued function $\Pi(t)$. The following result holds.

Theorem 2: Consider system (1) and the signal generator (6). Assume Assumptions 2 and 3 hold, $\sigma(A) \subset \mathbb{C}_{<0}$ and $\Lambda(t)$ is almost everywhere differentiable. Let

$$
\Pi(t)=\left(e^{A\left(t-t_{0}\right)} \Pi\left(t_{0}\right)+\int_{t_{0}}^{t} e^{A(t-\tau)} B L \Lambda\left(\tau, t_{0}\right) d \tau\right) \Lambda\left(t, t_{0}\right)^{-1}
$$

be a family of matrix valued functions parametrized in $\Pi\left(t_{0}\right) \in$ $\mathbb{R}^{n \times \nu}$. Then there exists a unique $\Pi_{\infty}\left(t_{0}\right)$ such that, for any $\Pi\left(t_{0}\right), \lim _{t \rightarrow+\infty} \Pi(t)-\Pi_{\infty}(t)=0$, where $\Pi_{\infty}(t)$ is the solution of (10) with $\Pi\left(t_{0}\right)=\Pi_{\infty}\left(t_{0}\right)$. Moreover, if $x\left(t_{0}\right)=$ $\Pi_{\infty}\left(t_{0}\right) \omega\left(t_{0}\right)$ then $x(t)-\Pi_{\infty}(t) \omega(t)=0$ for all $t \geq t_{0}$, and the set $\mathcal{M}_{\infty}=\left\{(x, \omega) \in \mathbb{R}^{n+\nu} \mid x(t)=\Pi_{\infty}(t) \omega(t)\right\}$ is attractive.

Proof: Let $\mathcal{T} \subset \mathbb{R}_{\geq 0}$ be a set in which $\Lambda\left(t, t_{0}\right)$ is differentiable with respect to $t$. Differentiating both sides of equation (10) over $\mathcal{T}$ yields

$$
\begin{gathered}
\dot{\Pi}(t) \Lambda\left(t, t_{0}\right)+\Pi(t) \dot{\Lambda}\left(t, t_{0}\right)-A e^{A\left(t-t_{0}\right)} \Pi\left(t_{0}\right)= \\
=B L \Lambda\left(t, t_{0}\right)+A \int_{t_{0}}^{t} e^{A(t-\tau)} B L \Lambda\left(\tau, t_{0}\right) d \tau= \\
=B L \Lambda\left(t, t_{0}\right)+A \Pi(t) \Lambda\left(t, t_{0}\right)-A e^{A\left(t-t_{0}\right)} \Pi\left(t_{0}\right) .
\end{gathered}
$$

Then, since Assumption 2 holds, we have

$$
\dot{\Pi}(t)=A \Pi(t)+B L-\Pi(t) \dot{\Lambda}\left(t, t_{0}\right) \Lambda\left(t, t_{0}\right)^{-1} .
$$

Let $\Pi_{1}(t)$ and $\Pi_{2}(t)$ be the solutions of equation (11) with initial conditions $\Pi_{1}\left(t_{0}\right)$ and $\Pi_{2}\left(t_{0}\right)$, respectively, and define the error $\hat{E}(t)=\Pi_{1}(t)-\Pi_{2}(t)$. Then

$$
\begin{aligned}
\dot{\hat{E}}(t)=A & \Pi_{1}(t)+B L-\Pi_{1}(t) \dot{\Lambda}\left(t, t_{0}\right) \Lambda\left(t, t_{0}\right)^{-1}- \\
& -\left(A \Pi_{2}(t)+B L-\Pi_{2}(t) \dot{\Lambda}\left(t, t_{0}\right) \Lambda\left(t, t_{0}\right)^{-1}\right)= \\
= & A \hat{E}(t)-\hat{E}(t) \dot{\Lambda}\left(t, t_{0}\right) \Lambda\left(t, t_{0}\right)^{-1}
\end{aligned}
$$

and (see [23, Section 11])

$$
\hat{E}(t)=e^{A\left(t-t_{0}\right)} \hat{E}\left(t_{0}\right) \Phi\left(t, t_{0}\right)^{\top} .
$$

Since $\Phi\left(t, t_{0}\right)^{\top}$ is bounded by a polynomial, by Assumption 3, $\hat{E}(t)$ converges to zero. This implies that there exists a motion $\Pi_{\infty}(t)$ to which all solutions of equation (11) converge, i.e. for any $\Pi\left(t_{0}\right)$ there exists a $\Pi_{\infty}\left(t_{0}\right)$ such that $\lim _{t \rightarrow+\infty} \Pi(t)-$ $\Pi_{\infty}(t)=0$. Moreover, $\Pi_{\infty}(t)$ is unique for any $t \geq t_{0}$ by the piecewise continuity of $\dot{\Lambda}\left(t, t_{0}\right) \Lambda\left(t, t_{0}\right)^{-1}$, see e.g. [27, Theorem 3.2].

By Assumption 2, the unique solution of system (1) with input $u$ defined by equation (6) is

$$
x(t)=e^{A\left(t-t_{0}\right)} x\left(t_{0}\right)+\int_{t_{0}}^{t} e^{A(t-\tau)} B L \Lambda\left(\tau, t_{0}\right) \omega\left(t_{0}\right) d \tau .
$$

Let $x\left(t_{0}\right)=\Pi_{\infty}\left(t_{0}\right) \omega\left(t_{0}\right)$, straightforward computations show that

$$
\begin{aligned}
& x(t)-\Pi_{\infty}(t) \omega(t)= \\
& e^{A\left(t-t_{0}\right)} \Pi_{\infty}\left(t_{0}\right) \omega\left(t_{0}\right)+\int_{t_{0}}^{t} e^{A(t-\tau)} B L \Lambda\left(\tau, t_{0}\right) \omega\left(t_{0}\right) d \tau- \\
& -\left(e^{A\left(t-t_{0}\right)} \Pi_{\infty}\left(t_{0}\right)+\int_{t_{0}}^{t} e^{A(t-\tau)} B L \Lambda\left(\tau, t_{0}\right) d \tau\right) \times \\
& \quad \times \Lambda\left(t, t_{0}\right)^{-1} \Lambda\left(t, t_{0}\right) \omega\left(t_{0}\right)=0,
\end{aligned}
$$

for all $t \geq t_{0}$. The attractivity of $\Pi_{\infty}(t)$ and the invariance of $x(t)=\Pi_{\infty}(t) \omega(t)$ imply that the set $\mathcal{M}_{\infty}$ is attractive.

Corollary 1: Under the assumptions of Theorem 2 and Assumption 1, the function $\Pi_{\infty}(t) \omega(t)$ is the steady-state response $x_{s}(t)$ of $x(t)$, i.e. for any $x\left(t_{0}\right)$ and $\omega\left(t_{0}\right), \lim _{t \rightarrow+\infty} x(t)-$ $\Pi_{\infty}(t) \omega(t)=0$.

Remark 3: The definition of the function $\Pi_{\infty}(t)$ can be given as in (10) or, alternatively, as the unique solution of

$$
\dot{\Pi}(t)=A \Pi(t)+B L-\Pi(t) \dot{\Lambda}\left(t, t_{0}\right) \Lambda\left(t, t_{0}\right)^{-1} .
$$

with the initial condition $\Pi\left(t_{0}\right)=\Pi_{\infty}\left(t_{0}\right)$. From a practical point of view to determine $\Pi_{\infty}(t)$ from equation (10) or (12) it is necessary to know the initial condition $\Pi_{\infty}\left(t_{0}\right)$. However, since the motion $\Pi_{\infty}(t)$ is attractive, any solution of the two equations converges to $\Pi_{\infty}(t)$, i.e. one could select $\Pi\left(t_{0}\right)=0$.

Remark 4: The integral equation (10) or the differential equation (12) play the role of the Sylvester equation (3). Unlike when we have an implicit model of the signal generator, the matrix $\Pi_{\infty}(t)$ is in general a function of time. In fact, as remarked in [22], infinitely many interpolation points arise whenever a periodic discontinuous signal is considered. Thus, a constant $\Pi$ should have infinitely many rows and columns.

Definition 2: Consider system (1) and the signal generator (6). Suppose Assumptions 1, 2 and 3 hold and $\sigma(A) \subset$ $\mathbb{C}_{<0}$. The function $C \Pi_{\infty}(t) \omega(t)$, where $\Pi_{\infty}(t)$ is the solution of equation (10) with $\Pi\left(t_{0}\right)=\Pi_{\infty}\left(t_{0}\right)$, is defined as the moment of system (1) at $\Lambda\left(t, t_{0}\right)$.

Corollary 2: Consider the interconnection of system (1) with the signal generator (6). Suppose Assumptions 1, 2 and 3 hold, $\sigma(A) \subset \mathbb{C}_{<0}$ and $L$ and $\omega\left(t_{0}\right)$ are such that $\sigma\left(\mathcal{L}\left(L \Lambda\left(t, t_{0}\right) \omega\left(t_{0}\right)\right)\right)=\sigma\left(\mathcal{L}\left(\Lambda\left(t, t_{0}\right)\right)\right)$. Then the moment of (1) at $\Lambda\left(t, t_{0}\right)$ coincides with the steady-state response of the output of the interconnected system (1)-(6).

Proof: By the hypothesis on $A$ and Assumptions 1, 2 and 3 the steady-state response of (1) is well-defined and the relation $x_{s}(t)=\Pi_{\infty}(t) \omega(t)$, where $\Pi_{\infty}(t)$ is the unique solution of (10) with $\Pi\left(t_{0}\right)=\Pi_{\infty}\left(t_{0}\right)$, holds. By Theorem 2 the set $\mathcal{M}_{\infty}$ is attractive and the steady-state response of the output of the interconnected system corresponds to $C \Pi_{\infty}(t) \omega(t)$, which by definition is the moment of the system.

The choice of defining the moment of (1) as in Definition 2 is justified by the equivalence, when an implicit model of (6) is available, between the new and the classical definition [12]. In the next result we prove that, under certain hypotheses, the solutions of the Sylvester equation (3) and of the integral equation (10) are the same.

Theorem 3: Consider the signal generator (2), suppose $\sigma(A) \subset \mathbb{C}_{<0}$ and let $\sigma(S) \subset \mathbb{C}_{\geq 0}$. Let the triple $\left(L, S, \omega\left(t_{0}\right)\right)$ 
be minimal. Then the unique solution of the integral equation (10) with $\Pi\left(t_{0}\right)=\Pi_{\infty}\left(t_{0}\right)$ coincides with the unique solution of the Sylvester equation (3).

Proof: Firstly note that Assumptions 1, 2 and 3 hold for the signal generator (2). Let $\tilde{\Pi}$ be the unique solution of the Sylvester equation $A \tilde{\Pi}+B L=\tilde{\Pi} S$ and $\Pi_{\infty}(t)$ be the unique solution of the integral equation (10) with $\Pi\left(t_{0}\right)=\Pi_{\infty}\left(t_{0}\right)$. Computing the derivative of the error $E(t)=\Pi_{\infty}(t)-\tilde{\Pi}$ yields

$$
\begin{aligned}
\dot{E}(t) & =A \Pi_{\infty}(t)-\Pi_{\infty}(t) S+B L-0= \\
& =A \Pi_{\infty}(t)-\Pi_{\infty}(t) S-(A \tilde{\Pi}-\tilde{\Pi} S)=A E(t)-E(t) S,
\end{aligned}
$$

and (see [23, Section 11])

$$
E(t)=e^{A\left(t-t_{0}\right)} E\left(t_{0}\right) e^{-S\left(t-t_{0}\right)},
$$

which implies that $\Pi_{\infty}(t)-\tilde{\Pi}$ converges to zero. Since $\tilde{\Pi}$ is constant and $\Pi_{\infty}(t)$ is the limiting solution of (10), it follows that $\Pi_{\infty}\left(t_{0}\right)=\tilde{\Pi}, E\left(t_{0}\right)=0$ and then $E(t)=0$ for all $t \geq t_{0}$, which proves the claim.

As anticipated in the previous section, we now focus our interest on periodic signals.

Corollary 3: Consider system (1) and the signal generator (6). Assume Assumptions 1, 2 and 3 hold. If for (6) the property

$$
\Lambda\left(t, t_{0}\right)=D(t) \Lambda\left(t-T, t_{0}\right), \quad t \geq T+t_{0},
$$

holds with $D(t) \in \mathbb{R}^{\nu \times \nu}$ non-singular for all $t \in \mathbb{R}_{\geq 0}$ and $T \in \mathbb{R}_{>0}$, then equation (10) becomes

$$
\begin{aligned}
& \Pi_{\infty}(t)=e^{A T} \Pi_{\infty}(t-T) D(t)^{-1}+ \\
& \quad+\left[\int_{t-T}^{t} e^{A(t-\tau)} B L \Lambda\left(\tau, t_{0}\right) d \tau\right] \Lambda\left(t-T, t_{0}\right)^{-1} D(t)^{-1} .
\end{aligned}
$$

If $D(t)=I$ then

$$
\Pi_{\infty}(t)=\left(I-e^{A T}\right)^{-1}\left[\int_{t-T}^{t} e^{A(t-\tau)} B L \Lambda\left(\tau, t_{0}\right) d \tau\right] \Lambda\left(t, t_{0}\right)^{-1}
$$

Proof: Equation (14) is obtained substituting

$$
x_{s}(t)=\Pi_{\infty}(t) \omega(t)=\Pi_{\infty}(t) D(t) \omega(t-T)
$$

and $\omega(t-T)=\Lambda\left(t-T, t_{0}\right) \omega\left(t_{0}\right)$ in

$$
x_{s}(t)=e^{A T} x_{s}(t-T)+\int_{t-T}^{t} e^{A(t-\tau)} B L \Lambda\left(\tau, t_{0}\right) \omega\left(t_{0}\right) d \tau .
$$

If $D(t) \equiv I, \omega(t)=\omega(t-T)$ and the steady-state of $x(t)$ is periodic with period $T$. Then $\Pi_{\infty}(t) \omega(t)=x_{s}(t)=x_{s}(t-$ $T)=\Pi_{\infty}(t-T) \omega(t-T)=\Pi_{\infty}(t-T) \omega(t)$ which implies that $\Pi_{\infty}(t)=\Pi_{\infty}(t-T)$ and equation (15) follows.

Remark 5: Equation (13) is a generalized form of equation (6) with the property (7) and describes a wide class of signals, possibly non-periodic. To show this note, for instance, that (2) can always be written as (13). In fact, for any $T \in \mathbb{R}_{\geq 0}$,

$$
\omega(t)=e^{S T} \omega(t-T), \quad \Lambda\left(t, t_{0}\right)=e^{S\left(t-t_{0}\right)} .
$$

Thus (2) can be described by (13) with $D=e^{S T}$ for all $t$.
For periodic signals equation (15) defines in a simple form the periodic matrix $\Pi_{\infty}(t)$. In addition, note that this definition of $\Pi_{\infty}(t)$ does not depend upon the initial condition. We refer the reader to [22] where the properties of $\Pi_{\infty}(t)$ are illustrated by means of examples.

\section{A FAMILY OF REDUCED ORDER MODEL}

In this section, exploiting the new definition of moment, a family of systems achieving moment matching is introduced and connections with the families of models given in [12] are drawn.

Definition 3: Consider system (1) and the signal generator (6). Suppose Assumptions 1, 2 and 3 hold, $\sigma(A) \in \mathbb{C}_{<0}$ and $L$ and $\omega\left(t_{0}\right)$ are such that $\sigma\left(\mathcal{L}\left(L \Lambda\left(t, t_{0}\right) \omega\left(t_{0}\right)\right)\right)=$ $\sigma\left(\mathcal{L}\left(\Lambda\left(t, t_{0}\right)\right)\right)$. Then the system described by the equations

$$
\begin{aligned}
& \xi(t)=F\left(t, t_{0}\right) \xi_{0}+\int_{t_{0}}^{t} G(t-\tau) u(\tau) d \tau, \\
& \psi(t)=H(t) \xi(t),
\end{aligned}
$$

with $\xi(t) \in \mathbb{R}^{\nu}, F\left(t, t_{0}\right) \in \mathbb{R}^{\nu \times \nu}, G(t) \in \mathbb{R}^{\nu \times \nu}$, is a model of system (1) at (6), if there exists a unique solution $P_{\infty}(t)$ of the equation

$$
P(t)=\left(F\left(t, t_{0}\right) P\left(t_{0}\right)+\int_{t_{0}}^{t} G(t-\tau) L \Lambda\left(\tau, t_{0}\right) d \tau\right) \Lambda^{-1}\left(t, t_{0}\right),
$$

with $P\left(t_{0}\right)=P_{\infty}\left(t_{0}\right)$ such that for any $P\left(t_{0}\right), \lim _{t \rightarrow+\infty} P(t)-$ $P_{\infty}(t)=0$ and

$$
C \Pi_{\infty}(t)=H(t) P_{\infty}(t),
$$

where $\Pi_{\infty}(t)$ is the unique solution of (10), with $\Pi\left(t_{0}\right)=$ $\Pi_{\infty}\left(t_{0}\right)$. System (16) is a reduced order model of system (1) at (6) if $\nu<n$.

Remark 6: In addition to the assumptions which guarantee the existence of the moment of system (1), we need two additional conditions for the existence of the reduced order model (16). Firstly the reduced order model has to be asymptotically stable, i.e. for each $\varepsilon>0$, there exists $\delta_{\varepsilon}>0$ such that if $\|\xi(0)\|<\delta_{\varepsilon}$ then $\|\xi(t)\|<\varepsilon$ and there exists a $\delta>0$ such that if $\xi(0)<\delta$ then $\lim _{t \rightarrow+\infty} \xi(t)=0$. This guarantees that the function $P_{\infty}(t)$ exists, i.e. the moment of system (16) exists. Secondly we need $P_{\infty}(t)$ to be non-singular for all $t \in \mathbb{R}_{\geq 0}$. This guarantees the existence of $H(t)$ for all $t \in \mathbb{R}_{\geq 0}$, i.e. the moments are matched.

We now discuss how to simplify the family of models (16) to achieve moment matching with additional constraints.

Property 1: ideally we would like to have $P_{\infty}(t)=I$ (as in [12] and [14]), which has proved to give a remarkable simplification in the definition of the reduced order model. We recall that the selection $P=I$ in [12] yields a family of systems, parametrized by a matrix $\Delta \in \mathbb{R}^{\nu}$, which is complete, i.e. the family contains all systems of dimensions $\nu$ achieving moment matching.

Property 2: at the same time we would like to bring the first equation of (16) to a form for which we can easily enforce additional constraints. This form is described by the selection

$$
F\left(t, t_{0}\right)=e^{\widetilde{F}\left(t-t_{0}\right)}, \quad G(t)=e^{\widetilde{F} t} \tilde{G},
$$


for some $\widetilde{F}$ and $\widetilde{G}$, which makes the first equation of (16) a representation in explicit form of a linear time-invariant system which has an implicit model.

However, differently from the family of reduced order models given in [12], it is not possible for the reduced order model (16) to satisfy both properties, namely having an implicit model and be such that $P_{\infty}(t)=I$. Note, for instance, that if $\omega(t)$ belongs to the class of signals satisfying (7) and we use the selection (19), then the steady-state $\xi_{s}(t)$ is periodic and so it is $P_{\infty}(t)$ (see the proof of Corollary $3)$. Thus, this first route in which we simplify the problem fixing $P_{\infty}(t)$ brings the problem of determining $F\left(t, t_{0}\right)$ and $G(t)$ from equation (17) (which may not be easy) and, at the same time, such that additional properties, e.g. stability, can be enforced on the reduced order model. As a second route we can fix $F\left(t, t_{0}\right)$ and $G(t)$ with the selection given in (19) which, however, brings the problem of finding $P_{\infty}(t)$ and solving equation (18) with respect to $H(t)$.

Both the choices are viable, however, it is easier to follow the second route, namely using the selection (19) and solving numerically (17) and (18). In this case the dynamics of the state $\xi$ can be described by a linear system in implicit form for which the solution to the problem of selecting $\widetilde{F}$ and $\widetilde{G}$ such that additional properties are satisfied is straightforward [12].

Remark 7: Both routes lead to models which are equivalent to the reduced order models given in [12] if $\Lambda\left(t, t_{0}\right)=$ $e^{S\left(t-t_{0}\right)}$. Following the first route easy computation shows that $F\left(t, t_{0}\right)=e^{(S-\Delta L)\left(t-t_{0}\right)}, G(t)=e^{(S-\Delta L) t} \Delta$, with $\Delta$ free, is a solution of equation (17) with $P_{\infty}(t)=I$. Following the second route, by Theorem 3, equation (17) is equivalent to the Sylvester equation associated to the general family of reduced order models given in [12].

Remark 8: As already discussed, if $\Lambda\left(t, t_{0}\right)=e^{S\left(t-t_{0}\right)}$ and $P_{\infty}(t)=P=I$, then in (19) $\widetilde{F}=S-\Delta L$ and $\widetilde{G}=\Delta$ with $\Delta$ free. The family of models (16) reduces to the family parametrized in $\Delta$ given in [12]. Note also that a different selection $\bar{P} \neq P=I$ yields the same class of models through a change of coordinates in the state space representation, namely $\widetilde{F}=\bar{P}(S-\Delta L) \bar{P}^{-1}, \widetilde{G}=\bar{P} \Delta, \widetilde{H}=C \Pi \bar{P}^{-1}$, and there is still only one free parameter $\Delta$. On the other hand, in the selection (19), we have two free parameters, namely $\widetilde{F}$ and $\widetilde{G}$ which can be totally independent of each other (this is possible since $H(t)$ in (18) is not constant).

We summarize these observations in the next statement in which we give a family of reduced order models in the case of periodic input signals.

Proposition 1: Consider system (1) and the signal generator (6) with the property (7). Suppose Assumptions 1, 2 and 3 hold, $\sigma(A) \in \mathbb{C}_{<0}$ and $L$ and $\omega\left(t_{0}\right)$ are such that $\sigma\left(\mathcal{L}\left(L \Lambda\left(t, t_{0}\right) \omega\left(t_{0}\right)\right)\right)=\sigma\left(\mathcal{L}\left(\Lambda\left(t, t_{0}\right)\right)\right)$. Then the system

$$
\begin{aligned}
& \dot{\xi}=\widetilde{F} \xi+\widetilde{G} u, \\
& \psi(t)=C \Pi_{\infty}(t) P_{\infty}(t)^{-1} \xi(t),
\end{aligned}
$$

with $\xi(t) \in \mathbb{R}^{\nu}, \widetilde{F} \in \mathbb{R}^{\nu \times \nu}, \widetilde{G} \in \mathbb{R}^{\nu \times \nu}$ and $\Pi_{\infty}(t)$ defined in
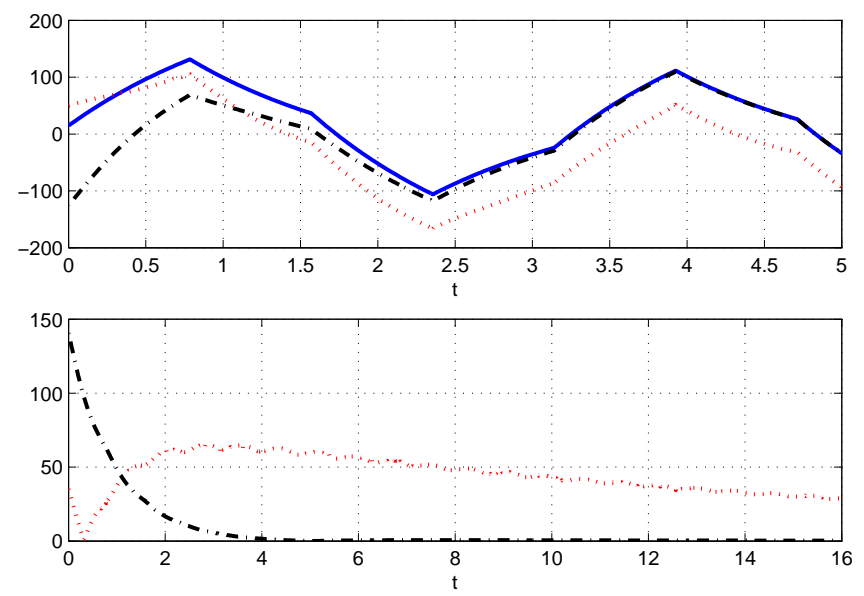

Fig. 1. Top: time history of the output $y$ (solid line) of system (1), with (22), of the output $\psi_{I}$ (dotted line) of system (20) and of the output $\psi_{I I}$ (dash-dotted line) of system (20) . Bottom: time history of $\left|y-\psi_{I}\right|$ (dotted line) and of $\left|y-\psi_{I I}\right|$ (dash-dotted line).

(15), is a model of system (1) at (6), if $\sigma(\widetilde{F}) \in \mathbb{C}_{<0}$ and

$$
P_{\infty}(t)=\left(I-e^{\widetilde{F} T}\right)^{-1}\left[\int_{t-T}^{t} e^{\widetilde{F}(t-\tau)} \widetilde{G} L \Lambda\left(\tau, t_{0}\right) d \tau\right] \Lambda\left(t, t_{0}\right)^{-1}
$$

is non-singular for all $t \in \mathbb{R}_{\geq 0}$.

Proof: In (16) select $F\left(t, t_{0}\right)$ and $G(t)$ as in (19). Since the signal generator (6) is periodic and $\sigma(\widetilde{F}) \in \mathbb{C}_{<0}$, equation (17) takes, by Corollary 3 the form given in (21). By assumption, $P_{\infty}(t)$ is non-singular and, thus, the matching condition (18) can be solved with respect to $H(t)$, giving $H(t)=C \Pi_{\infty}(t) P_{\infty}(t)^{-1}$.

We conclude this section with a numerical example to illustrate the results.

Example 1: Consider the interconnection of system (1) and (6). The matrices of (1), with $n=15$, have been selected as $A=\operatorname{diag}(-1 / n,-2 / n, \ldots,-1), B=$ $[-1 / n-2 / n \ldots-1]^{\top}, C=B^{\top}$, with the initial state $x\left(t_{0}\right)=\left[\begin{array}{llll}n / 2 & n / 2-1 & \ldots & -n / 2+1\end{array}\right]^{\top}$. Let $t_{0}=0$ and consider the matrix of square waves

$$
\Lambda_{\sqcap}(t, 0)=\left[\begin{array}{cc}
\sqcap\left(\frac{2 \pi}{T} t+\frac{\pi}{2}\right) & -\sqcap\left(\frac{2 \pi}{T} t\right) \\
\sqcap\left(\frac{2 \pi}{T} t\right) & \sqcap\left(\frac{2 \pi}{T} t+\frac{\pi}{2}\right)
\end{array}\right],
$$

where $\sqcap(t)=\operatorname{sign}(\sin (t))$, with $\operatorname{sign}(0)=0$, and $T=\pi$. Using system (20), two reduced order models of system (1) have been computed. The first one is described by the selection $\widetilde{F}=\operatorname{diag}(-1,-0.0667)$ (which are the slowest and fastest modes of $A$ ) and $\widetilde{G}=\left[\begin{array}{ll}0.0771-0.0251\end{array}\right]^{\top}$, while the second one by $\widetilde{F}=\operatorname{diag}(-0.9333,-1)$ (which are the two fastest modes of $A$ ) and $\widetilde{G}=\left[\begin{array}{ll}0.0891 & 0.008\end{array}\right]^{\top}$. In both case $G$ is such that $P_{\infty}(t)$ is non-singular for all $t \in \mathbb{R}_{\geq 0}$. The top graph of Fig. 1 shows the time history of the output $y$ (solid line), of the output $\psi_{I}$ of the first reduced order model (dotted line) and of the output $\psi_{I I}$ of the second reduced order model (dash-dotted line). The bottom graph shows the time history of $\left|y-\psi_{I}\right|$ (dotted line) and of $\left|y-\psi_{I I}\right|$ (dash-dotted line). 
The figure shows that the output response of the second model converges to the output of the system much quicker than the one of the first model.

Remark 9: The dynamics of (20) is the dynamics of a linear system in implicit form "filtered" with the dynamics of a linear system in explicit form.

Remark 10: Signals similar to the ones given in (22) can be generated by an implicit (nonlinear) model. In fact, note that the interconnection between the signals generated by the equations

$$
\dot{\omega}=\left[\begin{array}{cc}
0 & 1 \\
-1 & 0
\end{array}\right] \omega, \quad u=\operatorname{sign}\left(\left[\begin{array}{ll}
1 & 0
\end{array}\right] \omega\right),
$$

and system (1) can be studied with the nonlinear techniques proposed in [12]. However, in this case a partial differential equation has to be solved to compute the moment of the system and the general solution of this problem, possibly involving non-smooth functions, is difficult to find.

Remark 11: While from a computational point of view solving a partial differential equation and solving the integral and the matrix inversion in $\Pi_{\infty}(t)$ may be equally expensive, $\Pi_{\infty}(t)$ is an exact solution, while the solution of the partial differential equation would probably have to be approximated. Note also that, exploiting the periodicity of the steady-state, $\Pi_{\infty}(t)$ has to be computed only over a period. This can be done off-line and the obtained values can then be used on-line for any time interval.

Remark 12: Signals similar to the ones given in (22) can be also generated by hybrid systems. In fact, the signal generator (6) can represent a class of explicit linear hybrid systems. Thus, the results of the paper can be used to extend the model reduction method by moment matching to this class of hybrid systems.

\section{CONCLUSION}

The model reduction techniques based on moment matching have been extended to the class of input signal generators described by systems in explicit form. A time-varying parametrization of the steady-state of the system has been used to extend, using an integral matrix equation, the definition of moment to this class of input signals. The equivalence of the new definition and the one based on the Sylvester equation has been proved under specific conditions. Special attention has been given to periodic signals for the wide range of practical applications where these are used. Reduced order models matching the steady-state response of explicit signal generators have been given for linear systems and several connections between classical reduced order models and the new ones have been drawn. The paper is completed with a numerical example. We are currently working on an application of this methodology to extend the steady-state analysis of circuits to non-sinusoidal sources.

\section{REFERENCES}

[1] V. M. Adamjan, D. Z. Arov, and M. G. Krein, "Analytic properties of Schmidt pairs for a Hankel operator and the generalized Schur-Takagi problem," Mathematics of the USSR Sbornik, vol. 15, pp. 31-73, 1971.
[2] K. Glover, "All optimal Hankel-norm approximations of linear multivariable systems and their $\mathrm{L}^{\infty}$-error bounds," International Journal of Control, vol. 39, no. 6, pp. 1115-1193, 1984.

[3] M. G. Safonov, R. Y. Chiang, and D. J. N. Limebeer, "Optimal Hankel model reduction for nonminimal systems," IEEE Transactions on Automatic Control, vol. 35, no. 4, pp. 496-502, 1990.

[4] B. C. Moore, "Principal component analysis in linear systems: Controllability, observability, and model reduction," IEEE Transactions on Automatic Control, vol. 26, no. 1, pp. 17-32, 1981.

[5] D. G. Meyer, "Fractional balanced reduction: model reduction via a fractional representation," IEEE Transactions on Automatic Control, vol. 35, no. 12, pp. 1341-1345, 1990.

[6] W. S. Gray and J. Mesko, "General input balancing and model reduction for linear and nonlinear systems," in European Control Conference, Brussels, Belgium, 1997.

[7] A. C. Antoulas, J. A. Ball, J. Kang, and J. C. Willems, "On the solution of the minimal rational interpolation problem," Linear Algebra and Its Applications, Special Issue on Matrix Problems, vol. 137-138, pp. 511573, 1990.

[8] C. I. Byrnes, A. Lindquist, and T. T. Georgiou, "A generalized entropy criterion for Nevanlinna-Pick interpolation with degree constraint," IEEE Transactions on Automatic Control, vol. 46, pp. 822-839, 2001.

[9] S. Gugercin, A. C. Antoulas, and C. Beattie, " $\mathcal{H}_{2}$ model reduction for large-scale linear dynamical systems," SIAM Journal on Matrix Analysis and Applications, vol. 30, no. 2, pp. 609-638, 2008.

[10] K. Gallivan, A. Vandendorpe, and P. Van Dooren, "Sylvester equations and projection-based model reduction," Journal of Computational and Applied Mathematics, vol. 162, no. 1, pp. 213-229, 2004.

[11] A. Antoulas, Approximation of Large-Scale Dynamical Systems. Philadelphia, PA: SIAM Advances in Design and Control, 2005.

[12] A. Astolfi, "Model reduction by moment matching for linear and nonlinear systems," IEEE Transactions on Automatic Control, vol. 55 , no. 10 , pp. 2321-2336, 2010.

[13] T. C. Ionescu and A. Astolfi, "Families of reduced order models that achieve nonlinear moment matching," in Proceedings of the 2013 American Control Conference, Washington, DC, USA, June 17-19, 2013, pp. 5518-5523.

[14] G. Scarciotti and A. Astolfi, "Model reduction of neutral linear and nonlinear time-invariant time-delay systems with discrete and distributed delays," IEEE Transactions on Automatic Control, 2015.

[15] _ _ "Model reduction for linear systems and linear time-delay systems from input/output data," in 2015 European Control Conference, Linz, July, 2015.

[16] L. A. Zadeh and C. A. Desoer, Linear system theory: The state space approach, ser. McGraw-Hill series in System Science. McGraw-Hill, 1963.

[17] R. E. Kalman, P. L. Falb, and M. A. Arbib, Topics in mathematical system theory, ser. International series in pure and applied mathematics. McGraw-Hill, 1969.

[18] S. H. Wu, R. B. Sherwood, and R. Covey, "Block II Apollo digital reaction control systems study," TRW Space Technical Laboratory Report 373D-6004-RU000, June, 1965.

[19] A. Khayatian and D. G. Taylor, "Multirate modeling and control design for switched-mode power converters," IEEE Transactions on Automatic Control, vol. 39, no. 9, pp. 1848-1852, 1994.

[20] H. Sira-Ramirez and L. S. Orestes, "On the dynamical pulse-widthmodulation control of robotic manipulator systems," International Journal of Robust Nonlinear Control, vol. 6, no. 6, 1996.

[21] D. G. Taylor, "Pulse-width modulated control of electromechanical systems," IEEE Transactions on Automatic Control, vol. 37, no. 4, pp. 524-528, 1992.

[22] G. Scarciotti and A. Astolfi, "Characterization of the moments of a linear system driven by explicit signal generators," in Proceedings of the 2015 American Control Conference, Chicago, IL, July, 2015, pp. 589-594.

[23] R. W. Brockett, Finite dimensional linear systems, ser. Series in Decision and Control. Wiley, 1970

[24] O. M. Grasselli, L. Menini, and S. Galeani, Sistemi dinamici. Introduzione all'analisi e primi strumenti di controllo. Milano: Hoepli, 2008.

[25] A. Saberi, P. Sannuti, and A. Stoorvogel, Control of Linear Systems with Regulation and Input Constraints. London, UK: Springer-Verlag, 2000.

[26] B. A. Francis, "The linear multivariable regulator problem," in IEEE Conference on Decision and Control including the 15th Symposium on Adaptive Processes, Dec 1976, pp. 873-878.

[27] H. K. Khalil, Nonlinear Systems, 3rd ed. Englewood Cliffs: Prentice Hall, 2001. 